\title{
THE EFFECT OF IRRELEVANT FACTORS IN THE REFERENCE FIELD ON THE ACQUISITION OF A MINIATURE ARTIFICIAL LANGUAGE
}

\author{
KAZUO MORI ${ }^{1}$ \\ Institute for Research in Human Abilities, Memorial University of Newfoundland
}

\begin{abstract}
Three experiments with university students as subjects were carried out to examine the effect of irrelevant factors in the reference field on acquisition of a miniature artificial language. Despite presence of the irrelevant factors experimental subjects acquired the language with irrelevant factors as easily as subjects acquired without the irrelevant factors. In one of these experiments, Japanese-speaking subjects were used so that the results could be compared with a similar study using English-speaking subjects. Subjects having different native languages performed similarly in general, but showed a significant difference on the acquisition of a particular syntactic rule. The results are discussed from the viewpoints of the nature of reference situation and child language acquisition.
\end{abstract}

Several studies of language acquisition have used miniature artificial languages. These miniature artificial languages use verbal symbols as "words" and a set of rules as "syntax". Some miniature artificial languages also have "meanings" represented in several ways, such as by a set of typewriter operations (Miller \& Norman, 1964) or a set of visual objects (Moeser \& Bregman, 1972). Experimental subjects are required to learn a miniature artificial language under certain conditions, and their performance in ac-

1 A part of this study was done while the author, holder of the Toda Municipal Scholarship for 1978-79, was a predoctoral research fellow at the Institute for Research in Human Abilities, Memorial University of Newfoundland, Canada. I am much indebted to the Institute for offering the experimental facilities and the research expenses. I am also indebted to Dr. S. D. Moeser of the Institute for supervising this research. I especially wish to thank Paul Paschke, who did a superb job in editing my manuscript. Requests for reprints should be sent to Kazuo Mori, Institute for Research in Human Abilities, Memorial University of Newfoundland, St. John's, Newfoundland AlC 5 S7, Canada. quiring the miniature language is then compared with the natural language acquisition of children.

Although this is a typical methodology in experimental psychology, the validity of this experimental methodology as a research tool for the study of child language acquisition has been challenged by a number of researchers. Among these criticisms, the most crucial one is that these miniature artificial languages are too simple to adequately study the problems of natural language acquisition. (For the other criticisms and arguments on this topic, see Smith \& Braine, 1971; Moeser, 1977; Schlesinger, 1977). Moeser (1977) has answered this criticism by arguing that abstraction and simplification are the essence of laboratory experiments and should be considered an advantage. If true, then any miniature artificial language acquisition experiment has an essential dilemma. The experimental paradigm should be similar to the acquisition situation of natural language which is one of the most complex systems human beings have. Consequently, the more similar to natural language a mini- 
ature artificial language becomes, the more complex it must become. But at the same time it should be as simple as possible in order to control the relevant variables.

There seem to be at least two approaches to tackling this dilemma. One of the approaches is, as Moeser (1977) suggested, to use different kinds of miniature artificial languages depending upon what aspect of language acquisition should be studied in the experiment, that is, to introduce the complexity only up to a certain point where it is shown to be necessary. If for example, language word order is being investigated, the miniature artificial language will contain only a simple word order rule, or if selection restriction rules are being investigated, the miniature language syntax will be made more complex. In this way, the miniature language can reflect an aspect of the complexity of natural language, yet at the same time the entire system of the miniature language remains simple.

The other approach is to elaborate the artificial language through experiments. For example, if two artificial languages, one having an additional factor in its structure, are used in an experiment and they are shown to cause no differences, the additional factor is unnecessary and can be eliminated from further experiments while still retaining the same level of simulation. On the other hand, if this additional factor were proved to be important, it would be introduced to all the experimental paradigms thereafter. In this way, the experimental paradigm would contain more factors, but it would be a necessary complexity and it might be still simpler as a whole by reducing the complexity in unnecessary aspects.

Moeser and Bregman (1972) introduced a semantic factor - the referent-into the experimental paradigm of miniature artifcial language acquisition. They found that learning the complex features of syntax was possible only when these fea- tures reflected properties of the reference field. In their experimental design, a sentence of the miniature artificial language was coupled with a corresponding referent in the form of a geometric figure. The referents were organized in a certain way that was related to the grammatical structure of the artificial language. They found that subjects who were presented sentences of the artificial language with referents were able to acquire its grammatical structure whereas subjects presented only sentences were not. They also found that subjects presented sentences with referents used a strategy different from those presented only sentences. These findings are consistent with recent views on language acquisition in children presented by Schlesinger (1971) and others, that also stress the importance of the reference field.

Moeser and Bregman (1972), therefore, proved not only the importance of the reference field for language acquisition, but also that a miniature artificial language should consist of a semantic system as well as a syntactic system. Because it was found that subjects presented only sentences would be performing a task qualitatively different from children in the natural situation, the miniature artificial language with a semantic system is better simulation of the natural language acquisition situation than those without a semantic system.

In the experimental design of Moeser and Bregman (1972) a sentence was presented along with a corresponding referent. This seems at first thought a good simulation of the natural situation where a child learns his first language. However, in the natural situation, although a sentence and its referent are coupled, there is also some extraneous and irrelevant information that might obscure the correspondence between the sentence and its referent. That is, what a child actually sees when he hears a sentence such as, "A doggie is coming," is not just a dog 
by itself but, for example, a dog led by its owner or a dog chasing a cat. In any case, a child will see any referent along with its entire background. Children, as they acquire a linguistic structure, must find out what the referent is before they can use it. For this reason it cannot be concluded that the subjects in the Moeser and Bregman experiment utilized referents in the same way as children in a natural language acquisition situation. Rather, it might be argued that the experimental subjects acquired the artificial language only because they were provided with relevant referents. If this is the case, it is necessary to introduce some irrelevant factors, such as background, into the reference field of the experimental model in order to test whether subjects can acquire the artificial language under these conditions and to discover if they follow essentially the same acquisition process whether irrelevant factors are present or not. In this way, as stated above, the methodology of the miniature artificial language acquisition experiment could be further elaborated as well. This is the purpose of the present study.

However, in order to prove that this experimental methodology is a proper simulation of the natural language acquisition process, it is also important to show that it is language-independent. In other words, the simulation should not be distorted by the linguistic knowledge of the subjects. Moeser and Olson (1974), in using a similar experimental acquisition method with children of ages $3: 6-4: 6$ years, obtained the same results as those from an experiment with university students. This would tend to indicate the validity of this experimental methodology despite chronological differences in the linguistic knowledge of subjects. In the same way, it might be possible to assume the validity of this methodology over cross-linguistic differences of subjects, if subjects of different languages showed the same results. Testing this hypothesis is another purpose of the present study.

\section{EXPERIMENT I}

For the present experiment, three reference conditions were designed; two of them were the same as those used in Moeser and Bregman (1972) and another one was added so that effects of background on language acquisition could be examined. The effects were examined under two different syntactic complexities. Thus, there were six experimental groups consisting of ten subjects each. In addition, since they were Japanese-speaking subjects it would be possible to assess the findings of Moeser and Bregman (1972) from a cross-linguistic point of view.

\section{Method}

Description of the miniature artificial language. The language consisted of fourteen "words" and a "grammatical structure". Fourteen CVCs were used as " words" which were divided into four classes; three classes consisted of four words each and one class consisted of two words. These classes were characterized by their roles in the sentences, being roughly equivalent to the parts of speech of natural language. Each word had as its referent a geometric figure. Four types of referents were used so that each type would correspond to each class of words. They were designed to be incorporated into systematic patterns that would be reflected in the structure of the sentence. The words and referents were the same as those used in Moeser and Bregman (1972). The following sets of rewrite rules describe the "grammatical structures" of the systems used in Experiment I:

System 1

$$
\begin{aligned}
& \mathrm{S} \longrightarrow \mathrm{AP}+\mathrm{BP}+(\mathrm{CP}) \\
& \mathrm{AP} \longrightarrow \mathrm{A}+(\mathrm{D}) \\
& \mathrm{BP} \longrightarrow \mathrm{B}+(\mathrm{CP}) \\
& \mathrm{CP} \longrightarrow \mathrm{C}
\end{aligned}
$$

System 2 


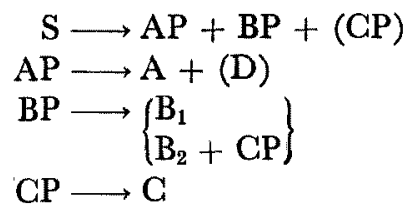

where $\mathrm{S}=$ sentence, $\mathrm{AP}=\mathrm{A}$-class word phrase, $\mathrm{BP}=\mathrm{B}$-class word phrase, $\mathrm{CP}=\mathrm{C}$-class word phrase, $\mathrm{A}=\mathrm{A}$ class word, $\mathrm{B}=\mathrm{B}$-class word, $\mathrm{B}_{1}$, $\mathrm{B}_{2}=$ sub-classes in B-class, $\mathrm{C}=\mathrm{C}$ class word, and $\mathrm{D}=\mathrm{D}$-class word.

These two systems differed in degree of complexity, so that it would be possible to examine the effect of background under two different syntactic complexities of the miniature artificial language. They were equivalent to the first two of the three systems used in Moeser and Bregman (1972).

Description of the reference conditions. There were three reference conditions in each language system under which subjects were tested: (a) Sentence-only, in which only the words of the language were
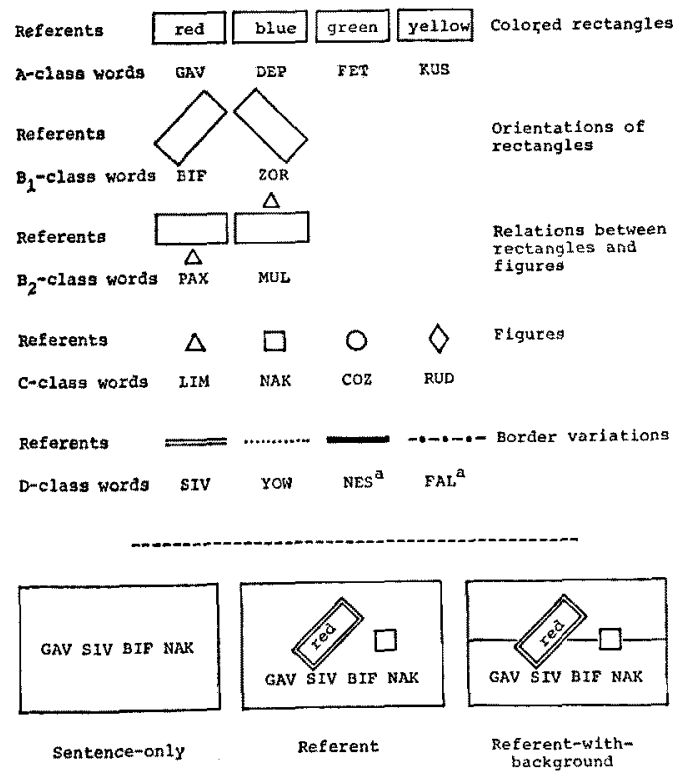

Fig. 1. Illustration of words and referents (above) and examples of presentation conditions (below).

a Used only in Experiments II \& III. presented in a sentence; (b) Referent, in which the referents were presented along with the sentences; (c) Referent-with-background, in which the referents were presented with a horizontal line randomly changed in color, either red, blue or green, along with the sentences. Conditions (a) and (b) were equivalent to the Words-only and Syntax correlation conditions of Moeser and Bregman (1972). An illustration of the words and referents is shown in Fig. 1 with examples of how one sentence would appear in each condition.

Procedure. For each system, 40 different sentences were used. Each word was presented 10 times in this group of 40 and all sentences were grammatically correct. Each presentation consisted of 80 sentences composed of two different series of the 40 sentences. The sentences were presented to subjects on slides projected on a screen by a Kodak 850 projector that automatically changed the slides every $6.5 \mathrm{~s}$ with a $0.8 \mathrm{~s}$ interval between slides. The instruction given to subjects was as follows: "This is a language learning experiment using an artificial language which refers to an artificial environment. The language has a grammatical structure which is not English or French or any other real language. It is your job to discover what this grammatical structure is. I will present this language to you on slides which will be shown on the screen in front of you. Above the words of the language are pictures to which the words refer. I want you to learn what each word refers to. (Referent and Referentwith-background conditions)" "This is a language learning experiment using an artificial language. The language has a grammatical structure which is not English or French or any other real language. It is your job to discover what this grammatical structure is. I will present this language to you on slides which will be shown on the screen in front of you. After a while you should see a systematic pattern in the way the words are arranged. 
I want you discover this pattern. (Sentence-only condition)" Subjects were given four presentations of the 80-slide series. After each presentation of 80 slides a test was given to measure the subjects' progress in learning the language. The tests were presented on paper and no figures appeared in the tests, only sentences. The four tests were identical in form but differed in terms of the sentences used. No sentence used in a test had previously been seen by the subjects. Each test contained three multiple-choice questions testing the following seven syntactic rules (for system 1, only rules 15 were used):

Rule (1) An A word must appear in a sentence.

Example: (a) GAV SIV ZOR RUD

(b) * LIM SIV ZOR RUD

Rule (2) Only one A word can appear in a sentence.

Example: (a) GAV PAX LIM

(b) *GAV KUS PAX LIM

Rule (3) A B word must appear in a sentence.

Example: (a) DEP PAX NAK

(b) *DEP NAK LIM

Rule (4) Only one B word can appear in a sentence.

Example:

(a) *KUS SIV BIF PAX RDU

(b) KUS SIV PAX RUD NAK

Rule (5) A D word can only follow an A word.

Example: (a) KUS YOW ZOR LIM

(b) *KUS ZOR YOW LIM

Rule (6) Two $\mathrm{C}$ words cannot appear with a $B_{1}$ word.

Example: (a) KUS BIF COZ

(b) *KUS BIF COZ NAK

Rule (7) A C word must appear with a $\mathrm{B}_{2}$ word.

Example: (a) GAV PAX RUD
(b) *GAV PAX

The sentences with asterisks are not grammatical.

Subjects. Subjects were 60 university students between the ages of 19 and 22,
TABLE 1

Experiment I : Means and standard deviations of the total correct scores

\begin{tabular}{lcr}
\hline Condition & Mean & \multicolumn{1}{c}{$S D$} \\
\hline System 1 & & \\
Sentence-only & 42.7 & 7.6 \\
& $47.2 \dagger$ & 4.4 \\
Referent & 53.3 & 12.1 \\
& 55.1 & 3.7 \\
Referent-with-BG & 56.0 & 4.8 \\
System 2 & & \\
Sentence-only & 60.6 & 6.0 \\
& 58.0 & 7.3 \\
Referent & 72.9 & 3.9 \\
& 78.9 & 4.2 \\
Referent-with-BG & 71.6 & 5.9 \\
Corresponding scores of & Moeser and Bregman \\
(1972) in italics. & &
\end{tabular}

attending an elementary psychology course at the University of Tsukuba, Japan. All were native speakers of Japanese. Ten subjects were assigned randomly to each group.

Results

The mean total correct scores on all four tests are shown in Table 1 and compared with the scores obtained by Moeser and Bregman (1972). All subjects in system 2 received 21 questions, whereas those subjects given system 1 had only 15 questions. These 15 questions in system 1 are, however, comparable to 15 questions in system 2 when the questions testing rules 6 and 7 are omitted. Thus the total $3 \times 2$ cell matrix consisting of the three referent conditions and two systems can be compared on the set of 15 questions that test rules 1 to 5 . A $3 \times 2$ analysis of variance indicated significant main effect due to the referent conditions $(F(2,54)=$ 16.72, $\left.M S_{\theta}=49.7, p<.01\right)$. The main effect due to the systems and the interaction were found not to be significant. Therefore, the five means obtained by the present experiment and by Moeser and Bregman (1972) in each system were 
TABle 2

Experiment I: Mean correct scores for the seven rules

\begin{tabular}{|c|c|c|c|c|c|c|c|}
\hline \multirow{2}{*}{ Condition } & \multicolumn{7}{|c|}{ Rule } \\
\hline & 1 & 2 & 3 & 4 & 5 & 6 & 7 \\
\hline \multicolumn{8}{|l|}{ System 1} \\
\hline \multirow[t]{2}{*}{ Sentence-only } & 10.1 & 8.9 & 7.9 & 6.8 & 9.0 & & \\
\hline & $10.6+$ & 11.0 & 7.9 & 7.9 & 9.8 & & \\
\hline \multirow[t]{2}{*}{ Referent } & 11.0 & 10.5 & 10.5 & 10.7 & 10.6 & & \\
\hline & 11.5 & 11.9 & 11.3 & 10.0 & 10.4 & & \\
\hline Referent-with-BG & 11.6 & 11.7 & 10.7 & 10.6 & 11.3 & & \\
\hline \multicolumn{8}{|l|}{ System 2} \\
\hline \multirow[t]{2}{*}{ Sentence-only } & 10.9 & 9.9 & 9.1 & 6.6 & 9.8 & 6.5 & 7.9 \\
\hline & 9.2 & 10.9 & 6.7 & 8.8 & 8.5 & 8.0 & 5.9 \\
\hline \multirow[t]{2}{*}{ Referent } & 11.7 & 11.6 & 10.9 & 10.7 & 11.5 & $7.6)$ & 8.9 \\
\hline & 11.5 & 11.6 & 11.0 & 11.0 & 11.5 & $11.7) *$ & 10.6 \\
\hline Referent-with-BG & 11.6 & 11.7 & 10.9 & 11.1 & 11.1 & 8.1 & 7.1 \\
\hline
\end{tabular}

+ Corresponding scores of Moeser and Bregman (1972) in italics.

* Significant difference at the 0.01 level.

analyzed by a one-way analysis of variance. In both systems, the analysis of variance showed over-all significances $(F(4,45)=$ $5.63, M S_{\theta}=57.8, p<.01$, for the system 1 condition; $F(4,45)=24.67, M S_{\mathrm{e}}=31.39, p$ $<.01$, for the system 2 condition).

Effect of background. For each system, the mean total correct score for the Referent-with-background condition was compared with the corresponding mean total correct scores for the Referent condition and the Sentence-only condition using the Scheffe's method. For both systems the subjects in the Referent-with-background condition learned the artificial language significantly better than those subjects in the Sentence-only condition. No significant differences were found between the Referent-with-background and Referent conditions. The mean correct scores for each rule of the Referent-withbackground groups under both systems were compared with the corresponding scores of the Referent groups (Table 2). There were no significant differences.

Cross-linguistic effect. The mean total correct scores and the mean correct scores for each rule obtained by the present experiment were compared with those obtained by Moeser and Bregman (1972). Although there was a similar over-all tendency in most scores between the two studies, a significant difference was found between the means on the rule 6 for the Referent groups in the system 2 condition. There was also a considerable difference between the means on the rule 7 for the Referent groups in the system 2 condition, although it was not significant. Consequently, these differences yielded the near-significant difference between the total correct scores for the Referent groups in the system 2 condition of the two studies.

\section{EXPERIMENT II}

In Experiment I, no differences were found between the Referent and Referentwith-background conditions. But in both these two conditions, the subjects got such high scores that there might be a ceiling effect. And this might have concealed the possible negative effect on the background. In addition, these high scores made it difficult to see clearly the learning 
processes. Thus, Experiment II was designed to examine the learning processes under three different reference conditions using a more complex linguistic system.

\section{Method}

Description of the miniature artificial linguistic system. The system used in this experiment is the same as system 3 of Moeser and Bregman (1972):

$$
\begin{aligned}
\mathrm{S} & \longrightarrow \mathrm{AP}+\mathrm{BP}+(\mathrm{CP}) \\
\mathrm{AP} & \longrightarrow \mathrm{A}+(\mathrm{D}) \\
\mathrm{BP} & \longrightarrow\left\{\begin{array}{l}
\mathrm{B}_{1} \\
\mathrm{~B}_{2}+\mathrm{CP}
\end{array}\right\} \\
\mathrm{CP} & \longrightarrow \mathrm{C}+(\mathrm{D})
\end{aligned}
$$

Two new CVCs were added to the vocabulary of the former study as D-class words, making this language even more difficult than the most difficult language system in Moeser and Bregman (1972).

Description of the reference conditions. There were three reference conditions; Sentence-only, Referent, and Referentwith-background. These conditions were identical to those of Experiment I, except that the Referent-with-background condition now used four colors (red, blue, green, and yellow) in a horizontal line as the background.

Procedure. The procedure was the same as that for Experiment I except that in the two conditions with referents, subjects were given an extra test at the end to measure how much vocabulary they acquired. This vocabulary test consisted of sixteen pictures which the subjects were required to describe by choosing one of four alternative words. The presentation time for each slide was shifted from $6.5 \mathrm{~s}$ to $10.0 \mathrm{~s}$, because subjects in pilot experiment, in which slides were presented every $6.5 \mathrm{~s}$, failed to acquire the language.

Subjects. Subjects were thirty students attending Memorial University of Newfoundland between the ages of 17 and 24 . All were native speakers of English. Ten subjects were assigned randomly to each group.

\begin{tabular}{|c|c|c|c|c|}
\hline \multirow{2}{*}{ Condition } & \multicolumn{2}{|c|}{ Rule Tests } & \multicolumn{2}{|c|}{$\begin{array}{c}\text { Vocabulary } \\
\text { Test }\end{array}$} \\
\hline & Mean & $S D$ & Mean & $S D$ \\
\hline Sentence-only & $53.1+$ & 7.0 & 一 & - \\
\hline Referent & 66.9 & 9.4 & $14.3 t t$ & 2.1 \\
\hline $\begin{array}{l}\text { Referent-with- } \\
\text { BG }\end{array}$ & 62.5 & 9.7 & 13.3 & 3.7 \\
\hline
\end{tabular}

\section{TABLE 3}

Experiment II : Mean correct scores on rule tests and vocabulary test

\section{Results}

Scores on the rule tests. The mean total correct scores on the rule tests for each condition are shown in Table 3. An analysis of variance revealed that the main effect due to the condition was significant $\left(F(2,27)=6.44, M S_{6}=77.16, p<.01\right)$. The scores for the Referent-with-background condition were compared with those corresponding scores for the Referent and Sentence-only conditions by the Scheffe's method. There was no significant difference between the Referent-with-background condition and the Referent condition. The difference between the scores for the Referent-with-background and Sentence-only conditions was not significant, although it approached significance.

Learning curves. The mean correct scores on each of the four rule tests for the three groups were plotted to illustrate the learning curves (Fig. 2). The learning

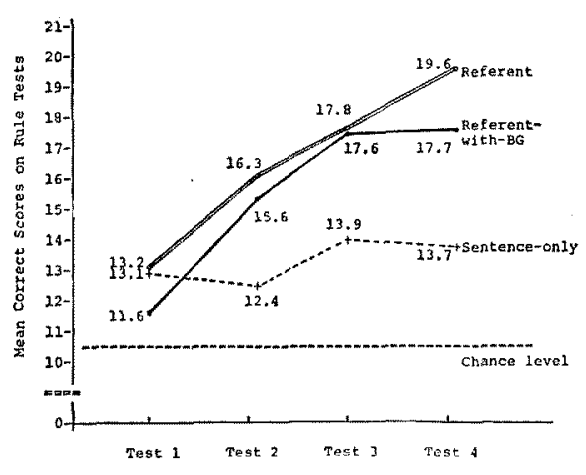

Frc. 2. Experiment II: Learning curves of the three groups. 
TABLE 4

Experiment III: Mean correct scores on rule tests

\begin{tabular}{|c|c|c|c|c|c|}
\hline \multirow{2}{*}{ Condition } & \multicolumn{5}{|c|}{ Test } \\
\hline & 1 & 2 & 3 & 4 & Total \\
\hline Referent & $12.2 \dagger$ & 13.8 & 14.6 & 17.8 & $58.4+\dagger$ \\
\hline Referent-with-irrelevant-figures & 10.8 & 13.0 & 15.6 & 16.6 & 56.0 \\
\hline
\end{tabular}

curve of the Sentence-only group differs greatly from the other two, while the latter two curves are of the same pattern. This suggests that subjects in the Referent and Referent-with-background conditions learn the artificial language in a similar way whereas in the Sentence-only condition, learning hardly occurs at all.

Scores on the vocabulary test. The vocabulary tests were given only to subjects in the Referent and Referent-with-background conditions. Mean correct scores for the vocabulary test are shown in Table 3 . Subjects in the Referent condition answered slightly better than did those in the Referent-with-background condition, but the difference was not significant. There were highly significant correlations between the vocabulary test scores and the rule test scores for both groups $(r=0.99$, $t=19.8, d f=8, p<.001$, for both groups). These correlations lend support to the hypothesis that the high performance of the subjects of both groups was accomplished mainly through the mediation of referents.

Oral reports from subjects. At the very end of the presentation in the Referentwith-background condition, subjects were asked whether or not they had figured out that the horizontal lines were irrelevant, and if so when. Seven subjects out of the ten answered that they figured out the irrelevance of the lines in the first presentation period. Two subjects answered that it was in the second period and the last subject did it in the third. No significant association $(p>.5)$ was found between whether or not they figured out this irrelevance in the first period and whether or not they acquired the language.

\section{EXPERIMENT III}

Before concluding that irrelevant factors in the reference field have little negative effect on language learning, one more point should be made. The irrelevant factors introduced in Experiments $I$ and II, horizontal lines, might not be perceptually distinct enough to have any effect. Experiment III was designed to examine further the effect of irrelevant factors in the reference field using different referent figures.

\section{Method}

Two new figures, which resembled the referents of the C-class words, were introduced as irrelevant factors. These two figures were a star-shape and a crushedsquare-shape. The size of the figures and the frequency of their appearance in the reference field were the same as that for other referents of the C-class words. All the other materials and procedures were identical to those of Experiment II, except that only two conditions--Referent and Referent-with-irrelevant-figures - were used. Subjects were ten students attending Memorial University of Newfoundland. All were native speakers of English.

\section{Results}

Mean total scores are shown in Table 4. Again, no significant differences were 
produced by the presence of the irrelevant factors.

\section{Discussion}

There seems no doubt that subjects in an experiment using an artificial language can eliminate irrelevant factors in a reference field and follow the same process as when no irrelevant factors are present in the reference field. This finding is consistent with the observation that children in a natural language acquisition situation seem to acquire the language despite a certain number of irrelevant factors in their reference field. Therefore this tends to indicate that child language acquisition can be simulated by an experimental artificial language acquisition model.

But why can irrelevant factors be eliminated so easily? One possible explanation might lie in the nature of the reference situation. The reference situation consists of one-to-one correspondences between symbols and referents. Suppose that symbols $a, b, c, d$, and their referents A, B, C, D are present at the same time. Each symbol has its own referent, and there are no irrelevant factors in this reference situation. But if each symbol (for example, a) and its referent (A) were considered separately in a one-to-one correspondence $(a-\mathrm{A})$, then the three other symbols $(b, c, d)$ and their referents $(B$, $C, D)$ would be, in a sense, irrelevant. If this reference situation were compared with another one in which the same symbols $(a, b, c, d)$ were present with their referents $(A, B, C, D)$ and with another factor (I) which had no corresponding symbol, then these two reference situations would only differ quantitatively. This was true for the two reference conditions in these experiments.

Moreover, although it was taken for granted that only relevant factors were present in the reference fields of the Referent conditions in the present experiments, this may be an unwarranted as- sumption. For instance, the shape of the rectangle was not described by any word ${ }^{2}$, nor were the horizontal relations between the small figures, whereas the vertical relations between the rectangle and the small figures were described by the $B_{2^{-}}$ class words. Consequently, both the Referent and Referent-with-background conditions contained some irrelevant factors. In other words, it was not only subjects in the Referent-with-background conditions, but also subjects in the Referent conditions who had to eliminate these irrelevant factors in order to acquire the language. The two conditions differed from each other only in the quantity of irrelevant factors present. This difference might have favored the subjects in the Referent conditions, but probably was not substantial enough to produce a significant difference in performance. This interpretation agrees with the experimental results. Although there were no statistically significant differences, the mean correct scores of the Referent-with-background groups tended to be lower than those of the Referent groups.

It could be argued that if a larger number of irrelevant factors were introduced in the reference field, eventually they would produce a nagative effect on the performance of subjects. But this case would likely never happen in an actual language acquisition situation, because a mother's speech to her child seems to be carefully selected to describe what he appears to perceive (cf. Newport, 1975; Brown, 1977). Therefore the number of irrelevant factors in the reference field is already limited within a certain range by mother's choice of words, and the child only needs to eliminate them. Consequently, there seems no need to introduce still more irrelevant factors to the miniature artificial language acquisition para-

2 Moeser and Bregman (1972) defined A-class words as colored rectangles, but the distinctive feature of the words was their color, not their form. 
digm. It will even be unnecessary to introduce any irrelevant factors for the sake of experimental "economy", since the present experiments have proved that introducing irrelevant factors will not make any difference in the essential capacity of the experimental paradigm to simulate accurately the natural language acquisition situation.

As for the cross-linguistic effect, the results from the experiment with Japanese subjects supported the major findings of Moeser and Bregman (1972) that subjects who learned an artificial language with referents were able to acquire its grammatical structure, whereas subjects presented only sentences were not. However, Japanese subjects who learned the artificial language with referents in Experiment I had more difficulty acquiring rules 6 and 7 than did their English-speaking counterparts who learned the same artificial language under the same conditions. This difference might be caused by the fact that in English the rules for the usage of transitive and intransitive verbs, to which rules 6 and 7 apply, are more strict than in Japanese where the frequent ellipsis of words obscures the existing rules. If that is the case, then this miniature artificial language would not be languageindependent in this aspect.

In conclusion, the present experiments have shown two things. First, subjects can acquire a language despite a certain number of irrelevant factors in the reference field. This result is compatible with observations of the natural language acquisition situation where children seem to acquire the language despite a certain number of irrelevant factors in their reference field. Second, subjects with differing linguistic knowledge acquire the language similarly as a whole, but differently as regards a particular syntactic rule.
These results tend to support the use of the miniature artificial language methodology for studying child language acquisition, but at the same time they suggest a limitation in using this methodology.

\section{REFERENCES}

Brown, R. 1977 Introduction, In C. Snow \& C. Ferguson (Eds.) Talking to thildren: Language and acquisition. Cambridge: Cambridge University Press. Pp. 1-27.

Miller, G. A., \& Norman, D. A. 1964 Research on the use of formal languages in the behavioral sciences. Semi-annual Technical Report, Department of Defence, Advanced Research Projects Agency. (Reported in G. A. Miller, 1967, The psychology of communication. New York: Basic Books.)

Moeser, S. D. 1977 Semantics and miniature artificial languages. In J. Macnamara (Ed.) Language learning and thought. New York: Academic Press. Pp. 227-250.

Moeser, S. D., \& Bregman, A. S. 1972 The role of reference in the acquisition of a miniature artificial language. Journal of Verbal Learning and Verbal Behavior, 11, 759-769.

Moeser, S. D., \& Olson, A. J. 1974 The role of reference in children's acquisition of a miniature artificial language. Journal of Experimental Child Psychology, 17, 204-218.

NewPort, E. L. 1975 Motherese: The speech of mothers to young children. Technical Report No. 52, Center for Human Information Processing. San Diego: University of California.

Schlesinger, I. M. 1971 Production of utterances and language acquisition. In D. I. Slobin (Ed.) The ontogenesis of grammar: $A$ theoretical symposium. New York: Academic Press. Pp. $63-101$.

Schlesinger, I. M. 1977 Miniature artificial languages as research tools. In J. Macnamara (Ed.) Language learning and thought. New York: Academic Press. Pp. 251-260.

Smith, K. H., \& Braine, M.D.S. 1971 Miniature languages and problem of language acquisition. Unpublished paper, Psychology Department, Bowling Green State University.

(Received Sep. 10, 1979) 